\title{
Impact of variation in physical activity after total joint replacement
}

\author{
Kazuhiro Hayashi ${ }^{1-3}$ \\ Masato Kako ${ }^{3}$ \\ Kentaro Suzuki ${ }^{3}$ \\ Yui Takagi ${ }^{3}$ \\ Chiaki Terai ${ }^{3}$ \\ Shotaro Yasuda ${ }^{3}$ \\ Izumi Kadono ${ }^{3}$ \\ Taisuke Seki ${ }^{4}$ \\ Hideki Hiraiwa ${ }^{4}$ \\ Takahiro Ushida' \\ Yoshihiro Nishida ${ }^{3,4}$ \\ 'Multidisciplinary Pain Center, Aichi \\ Medical University, Nagakute, Japan; \\ ${ }^{2}$ Department of Rehabilitation, Aichi \\ Medical University Hospital, Nagakute, \\ Japan; ${ }^{3}$ Department of Rehabilitation, \\ Nagoya University Hospital, Nagoya, \\ Japan; ${ }^{4}$ Department of Orthopaedic \\ Surgery, Nagoya University Graduate \\ School and School of Medicine, \\ Nagoya, Japan
}

This article was published in the following Dove Press journal: Journal of Pain Research

Purpose: Patients who undergo total knee arthroplasty (TKA) or total hip arthroplasty (THA) often develop postoperative pain. Exercise approaches are recommended postoperatively; however, the impact of excessive variation in physical activity is unclear. The purpose of the present preliminary study was to investigate the impact of excessive variation in physical activity using the accelerometer in the early period after TKA or THA.

Patients and methods: Seventy-two patients were enrolled in the study. Forty patients underwent initial TKA, and 32 initial THA. Physical activity was measured for 8 days from postoperative day 3 to 10 . Patients with substantial correlation between physical activity and postoperative day were classified as the "good-pacing" group. Patients with no correlation between them were classified as the "poor-pacing" group. They were also evaluated using a pain visual analog scale (VAS), pain catastrophizing scale, and hospital anxiety and depression scale.

Results: The average age was 68 years, and 59 patients ( $82 \%$ ) were women. The average maximum number of steps per day was 2,181 . There were 45 patients with good pacing and 27 with poor pacing. The poor-pacing group showed significantly lower maximum number of steps per day, higher postoperative average VAS score, higher postoperative worst VAS score, and longer duration of postoperative hospital stay than the good-pacing group.

Conclusion: Patients with excessive variation in physical activity showed severe postoperative pain and prolonged postoperative hospital stay. The postoperative variation in physical activity could be an outcome for improvement in patients after lower-limb arthroplasty.

Keywords: total joint arthroplasty, postoperative pain, rehabilitation, pacing, accelerometer

\section{Introduction}

Osteoarthritis is one of the most common types of arthritis of the knee and hip. Total knee arthroplasty (TKA) and total hip arthroplasty (THA) are treatment procedures for knee and hip osteoarthritis to reduce pain and disability and improve the functional status and quality of life. ${ }^{1}$ By 2030 , the incidence of THA and TKA is predicted to increase by approximately $174 \%$ and $673 \%$, respectively. ${ }^{1}$ Nevertheless, postoperative pain after TKA and THA often persists for many years. ${ }^{2}$ Postoperative pain interferes with rehabilitation, resulting in prolonged or incomplete recovery.

The regulation of physical activity is one of the nonpharmacological treatment options for pain. ${ }^{3,4}$ Previously, bed rest was a common treatment approach for acute musculoskeletal pain; however, more recently, exercise, as possible, has been recommended to improve pain and quality of life. ${ }^{4}$ In patients who underwent TKA and THA, early postoperative exercise, which involves getting out of bed and walking as
Multidisciplinary Pain Center, Aichi Medical University, I-I Yazakokarimata, Nagakute, Aichi 480-1 195, Japan

Tel/fax +8I 56 I62 5004

Email hayashi.kzhr@gmail.com 
soon as possible after the operation, is also recommended..$^{5-7}$ Increased postoperative physical activity is important in functional recovery. ${ }^{8,9}$

Subsequently, appropriate physical activity pacing is reported to be effective in reducing the severity of musculoskeletal pain. ${ }^{3}$ Physical activity pacing aimed to eliminate the variation of highs and lows in the activity patterns, keep a steady pace throughout the day, and avoid blind increase in physical activity., ${ }^{3,10}$ Poor physical activity pacing is associated with an increase in pain, psychological disturbances, and physical disability in patients with chronic pain. ${ }^{10}$ However, postoperative patients are often traditionally advised only to increase physical activity, as possible, and improve postoperative physical function. ${ }^{9}$ The impact of variation in physical activity throughout the day has not been investigated in postoperative patients.

The variation in physical activity is often assessed using the SD from the reference value. ${ }^{11-13}$ Some study investigated chronic pain ${ }^{11}$ and osteoarthritis for more than $3^{12}$ and 6 months after surgery in stable patients. ${ }^{13}$ In contrast, patients in the early postoperative period recover rapidly. The reference value of postoperative physical activity increases day by day. The high $\mathrm{SD}^{11-13}$ and low correlation coefficient ${ }^{14,15}$ values suggest a large variation from the reference value, both calculated by the least square method. Hence, we assessed variation in physical activity using the degree of correlation coefficient of physical activity and postoperative day (POD) for each subject.

The present preliminary study aimed to investigate the impact of variation in physical activity in the early period after TKA or THA. It is hypothesized that the variation in physical activity is associated with pain, and is consistent with that observed for patients with other types of musculoskeletal pain., ${ }^{3,10}$ Therefore, patients with excessive variation in physical activity are hypothesized to develop more severe postoperative pain than those with a gradual increase in physical activity.

\section{Patients and methods Participants}

The number of subjects was determined by a sample size estimation using $\mathrm{G}^{*}$ Power software (version 3.0.10; Franz Faul, Kiel University, Kiel, Germany). On the basis of a standardized effect size of $0.6, \alpha$-level of 0.05 , and power $(1-\beta)$ of 0.80 , the minimum number of subjects was estimated to be 72 .

This longitudinal cohort study enrolled a total of 72 participants. Forty patients underwent initial TKA, and 32 initial
THA between July 2015 and September 2016 at Nagoya University Hospital. The data for baseline demographics and in-hospital variables were obtained. The exclusion criteria were: 1) cognitive impairment and 2) pain in other body parts that was more severe than that in the operative site. All participants had a baseline preoperative visit prior to their operation and received standardized in-participant treatment, including usual rehabilitation (6 days/week), following either a primary total hip or total knee care pathway. Participants could receive nonsteroidal anti-inflammatory drugs for any painful condition.

This study was approved by the Ethics Committee of Nagoya University Hospital. All participants provided written informed consent.

\section{Measures}

\section{Demographic data}

Demographic data, including age, sex, height, body mass index, and other comorbidities using the Charlson comorbidity index ${ }^{16}$ were recorded. Intraoperative data, such as surgical procedure, operative time, and intraoperative blood loss were also collected. In addition, postoperative length of hospital stay was recorded.

\section{Evaluation of the physical activity}

All patients used the accelerometer $24 \mathrm{~h} /$ day in the hospital, except when bathing or sleeping at night. All patients wore the accelerometer on a belt at the waist level just above either leg. Indices were estimated with the accelerometer (Lifecorder GS; Suzuken, Nagoya, Japan), which provides reliable and validated output data. ${ }^{17,18}$ The daily total number of steps served as the index of physical activity. The patients and medical staff could look at the number of steps any time.

The measurement period was 8 days from PODs 3-10. The reason was the patients wore a one-piece hospital gown that obstructs the use of the accelerometer until POD 2, and they attained improvement to be discharged home as early as POD 11. After the measurement period, the device was retrieved, and the data were downloaded into a computer.

Patients with a substantial correlation $(P$-value $<0.05$ and correlation coefficient $>0.7$ ) between physical activity per day and during POD, as determined by single regression test, were classified as the "good-pacing group." Patients with no correlation $(P$-value $\geq 0.05)$ between them were classified as the "poor-pacing group." ${ }^{14,15}$ The changes in the physical activity of a case are shown in Figure 1, that is, a case in the good-pacing group showed that as more PODs passed, the greater was the physical activity per day. A case in the poor- 


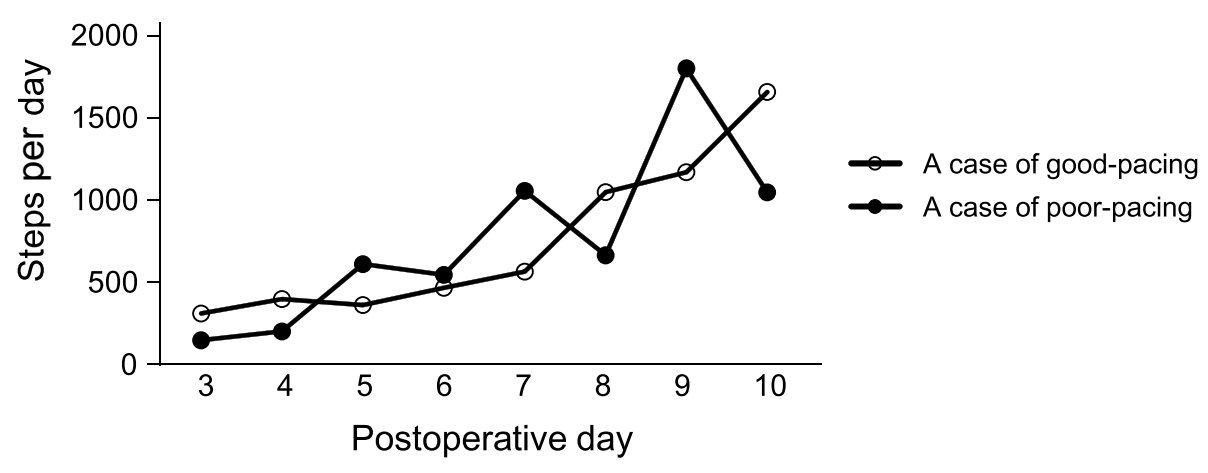

Figure I Changes in the physical activity of an instance case.

pacing group showed an increase or decrease in physical activity throughout the day.

\section{Pain and psychological questionnaire}

The patients rated the pain in the surgical site using a visual analog scale (VAS) for daily life activities. The participants were evaluated on admission. They were then evaluated on POD 14 or prior to hospital discharge (mean, 13.8 days after surgery).

The pain catastrophizing scale (PCS) score was measured on admission to assess the negative thought patterns about pain. ${ }^{19,20}$ For the 13-item PCS, participants rated how frequently they experienced various cognitions or emotions. ${ }^{19,20}$ The PCS consists of three subscales: rumination (eg, "I keep thinking about how much it hurts"), magnification (eg, "I wonder whether something serious may happen"), and helplessness (eg, "There is nothing I can do to reduce the intensity of the pain"). ${ }^{19,20}$ The total score range is $0-52 . .^{19,20}$ Several findings support this scale's validity as a measure of pain catastrophizing. ${ }^{19,20}$

Psychological characteristics were measured by the hospital anxiety and depression scale (HADS) on admission..$^{21,22}$ The HADS is designed to assess two separate dimensions of anxiety and depression. It consists of 14 items, with the anxiety (HADS-A) and depression (HADS-D) subscales each including seven items. A 4-point response scale (from 0 , representing absence of symptoms, to 3 , representing maximum symptoms) is used, with possible scores for each subscale ranging from 0 to $21 .{ }^{21,22}$ The HADS is designed to avoid the use of somatic symptoms that may confound other self-report measures of depression and anxiety. ${ }^{21,22}$

\section{Statistical analysis}

All continuous variables were expressed as the mean with the SD. First, patients were categorized into two groups: good-pacing and poor-pacing groups. Differences between the two groups were analyzed using the chi-squared test for categorical variables and the unpaired $t$-test for continuous variables. The data were analyzed using SPSS (version 24.0 for Microsoft Windows; SPSS, Chicago, IL, USA). A $P$-value $<0.05$ was considered statistically significant.

\section{Ethical approval}

This study was approved by the Ethics Committee of Nagoya University Hospital (No. 2015-0171).

\section{Results \\ Patient characteristics}

Patient characteristics are shown in Table 1. The average age was 68 years. The study included 13 men and 59 women. The preoperative scores for TKA, THA, and overall were 37.9, 44.2, and 40.7, respectively, for average VAS and 55.5, 57.6, and 56.4, respectively, for worst VAS. The postoperative scores for TKA, THA, and overall were 19.4, 16.1, and 17.9, respectively, for average VAS and 38.9, 27.8, and 34.0, respectively, for worst VAS. Figure 2 shows the group analysis of physical activity according to the surgical site. It shows that as more PODs passed, the greater was the physical activity in both TKA and THA.

The number of patients with good pacing in TKA, THA, and overall was $24(60 \%), 21(65 \%)$, and $45(62 \%)$, respectively. The number of patients with poor pacing in TKA, THA, and overall was $16(40 \%), 11$ (34\%), and $27(37 \%)$, respectively. There were no patients with slight or moderate correlation between steps and POD.

\section{Physical activity}

Differences in the pre-, intra-, and postoperative variables between the good- and poor-pacing groups are shown in Table 2. Among the overall preoperative variables, the poor-pacing group reported shorter height and more women than the good- 
Table I Patients' characteristics

\begin{tabular}{|c|c|c|c|}
\hline & $\begin{array}{l}\text { Overall } \\
(n=72)\end{array}$ & $\begin{array}{l}\text { TKA } \\
(n=40)\end{array}$ & $\begin{array}{l}\text { THA } \\
(n=32)\end{array}$ \\
\hline \multicolumn{4}{|l|}{ Preoperative data } \\
\hline Female gender, $\mathrm{n}(\%)$ & $59(82 \%)$ & $34(85 \%)$ & $25(78 \%)$ \\
\hline Age (years) & $69.0(10.5)$ & $72.4(7.4)$ & $64.7(12.4)$ \\
\hline Height $(\mathrm{cm})$ & I54.8 (7.8) & $153.3(6.6)$ & $156.6(10.6)$ \\
\hline BMI $\left(\mathrm{kg} / \mathrm{m}^{2}\right)$ & $24.8(4.1)$ & $26.1(3.9)$ & $23.2(4.0)$ \\
\hline $\begin{array}{l}\text { Charlson comorbidity } \\
\text { index }(0 / 1 / 2)\end{array}$ & $(44 / 23 / 5)$ & $(22 / 15 / 3)$ & $(22 / 8 / 2)$ \\
\hline Disease onset (months) & $73.8(72.3)$ & $89.7(74.4)$ & $53.8(65.2)$ \\
\hline Previous surgeries, $n$ (\%) & $20(27 \%)$ & II (27\%) & $9(28 \%)$ \\
\hline Pain in other joints, $n$ (\%) & $27(37 \%)$ & $18(45 \%)$ & $9(28 \%)$ \\
\hline Average VAS $(\mathrm{mm})$ & $40.7(21.9)$ & $37.9(20.0)$ & $44.2(23.9)$ \\
\hline Worst VAS (mm) & $56.4(26.8)$ & $55.5(26.8)$ & $57.6(27.1)$ \\
\hline HADS-anxiety (points) & $5.5(3.6)$ & $5.2(3.5)$ & $6.0(3.8)$ \\
\hline $\begin{array}{l}\text { HADS-depression } \\
\text { (points) }\end{array}$ & $5.6(3.4)$ & $5.4(3.0)$ & $5.8(3.8)$ \\
\hline PCS (points) & $25.6(12.7)$ & $23.4(12.8)$ & $28.4(12.2)$ \\
\hline \multicolumn{4}{|l|}{ Intraoperative data } \\
\hline Operative time (min) & $108(33)$ & $|2|(23)$ & $92(37)$ \\
\hline Blood loss (ml) & $211(4 \mid 9)$ & $51(64)$ & $413(568)$ \\
\hline \multicolumn{4}{|l|}{ Postoperative data } \\
\hline \multicolumn{4}{|l|}{$\begin{array}{l}\text { Correlation steps with } \\
\text { postoperative day, n (\%) }\end{array}$} \\
\hline $\begin{array}{l}\text { - no correlation (poor- } \\
\text { pacing) }\end{array}$ & 27 (37\%) & $16(40 \%)$ & II (34\%) \\
\hline - slight correlation & $0(0 \%)$ & $0(0 \%)$ & $0(0 \%)$ \\
\hline - moderate correlation & $0(0 \%)$ & $0(0 \%)$ & $0(0 \%)$ \\
\hline $\begin{array}{l}\text { - substantial correlation } \\
\text { (good-pacing) }\end{array}$ & 45 (62\%) & $24(60 \%)$ & $21(65 \%)$ \\
\hline Maximum steps & 2181 & 1925 & $2500(1920)$ \\
\hline (steps/day) & $(1803)$ & $(1684)$ & \\
\hline Average VAS (mm) & $17.9(14.3)$ & $19.4(14.2)$ & I6.I (14.5) \\
\hline Worst VAS (mm) & $34.0(25.2)$ & $38.9(25.9)$ & $27.8(23.2)$ \\
\hline Length of hospital (days) & $19.5(4.8)$ & $20.7(4.5)$ & $18.0(4.6)$ \\
\hline
\end{tabular}

Note: All continuous variables were expressed as mean and standard deviation. Abbreviations: TKA, Total Knee Arthroplasty; THA, Total Hip Arthroplasty; BMI, Body Mass Index; VAS, Visual Analog Scale; HADS, Hospital Anxiety and Depression Scale; PCS, Pain Catastrophizing Scale.

pacing group. Preoperative VAS, PCS, and HADS scores were not significantly different between the groups. Among the postoperative variables, the poor-pacing group showed significantly lower maximum steps per day, higher postoperative average VAS score, higher postoperative worst VAS score, and longer postoperative hospital stay than the good-pacing group.

\section{Discussion}

The aim of the present study was to investigate the impact of variation in physical activity in postoperative patients. The present study is the first to indicate that patients with variation in physical activity develop severe postoperative pain with a prolonged postoperative hospital stay. The variation

\section{A}

TKA

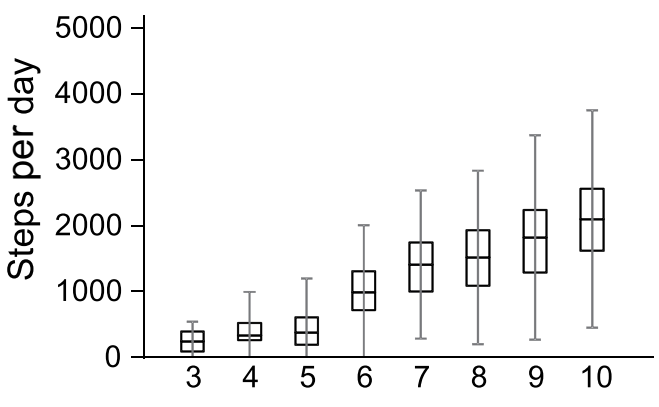

Postoperative day

B

THA

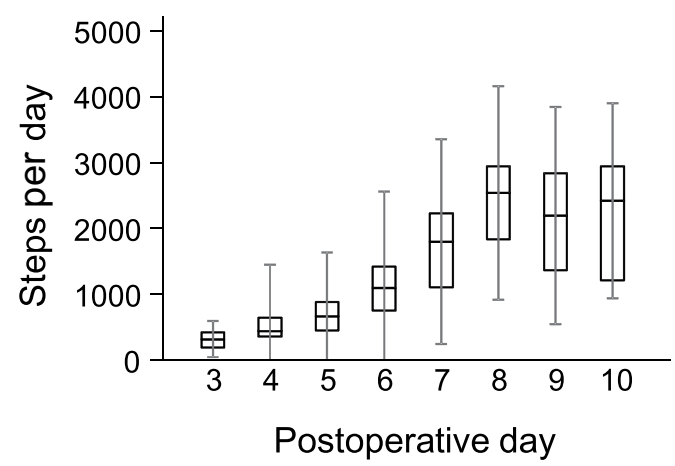

Figure 2 As more PODs passed, the physical activity increased. Notes: (A) TKA patients. (B) THA patients.

Abbreviations: POD, postoperative day; THA, total hip arthroplasty; TKA, total knee arthroplasty.

in physical activity in postoperative patients could be an outcome for improvement.

Variations in physical activity are associated with increased pain, psychological disturbances, and physical disability. ${ }^{10}$ The present study showed that a poor pacing during physical activity is associated with severe pain in postoperative patients, consistent with that observed for patients with other types of musculoskeletal pain. ${ }^{3,10}$ Overactivity results in a number of potential injuries to muscle fibers, nerves, bones, and ligaments. These injuries, as well as the repetitive experience of pain, will prolong pain and contribute to neurobiological mechanisms of peripheral and central sensitization. ${ }^{23}$ Moreover, underactivity is a result of fear of pain and avoidance of activity and prolongs pain, leading to higher levels of disability, according to the fearavoidance model. ${ }^{24}$ Activity pacing is effective in variation in physical activity in patients with chronic pain and osteoarthritis with overactivity and underactivity cycles., ${ }^{3,10,12}$ Till date, the effects of activity pacing have not been investigated in postoperative patients. 


\begin{tabular}{|c|c|c|c|c|c|}
\hline & 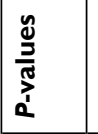 & 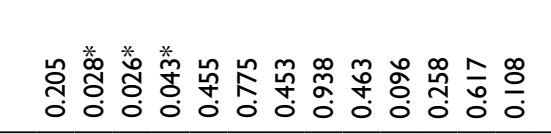 & 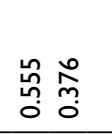 & 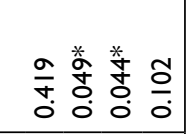 & \\
\hline & 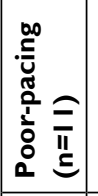 & 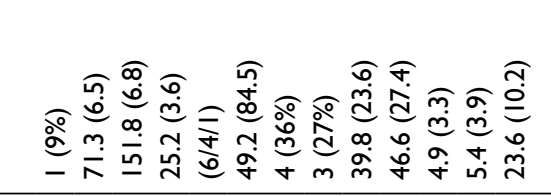 & 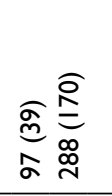 & 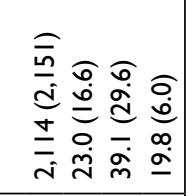 & \\
\hline$\underset{\mathbf{T}}{\mathbf{T}}$ & 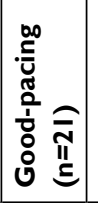 & 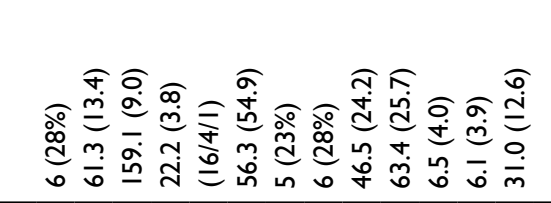 & 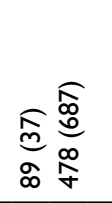 & 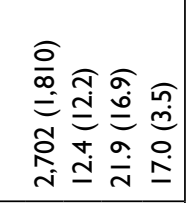 & \\
\hline & 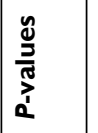 & 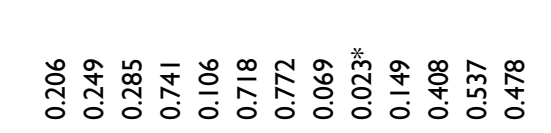 & 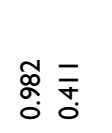 & 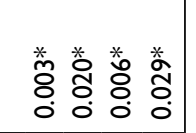 & \\
\hline & 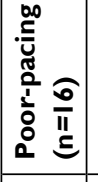 & 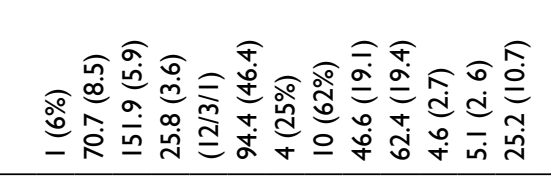 & 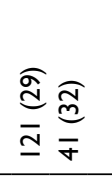 & 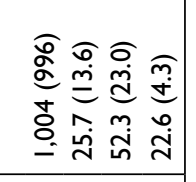 & \\
\hline$\underline{\mathbb{s}}$ & 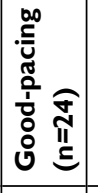 & 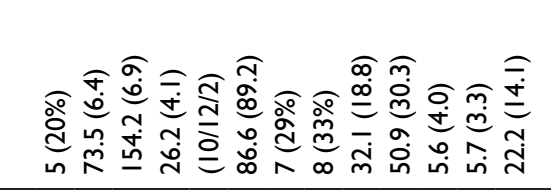 & 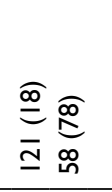 & 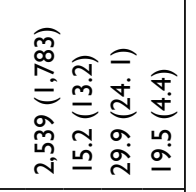 & \\
\hline & 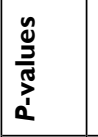 & 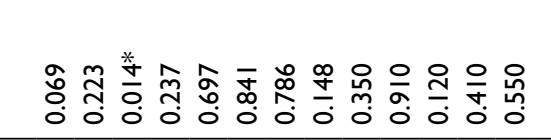 & 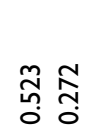 & 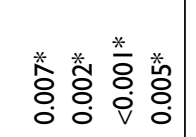 & \\
\hline & 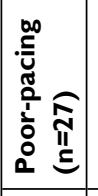 & 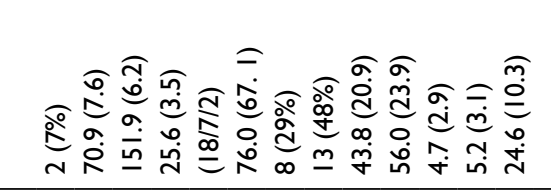 & 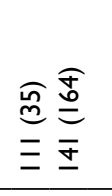 & 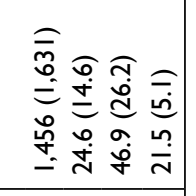 & 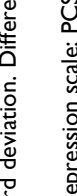 \\
\hline ర) & 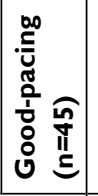 & 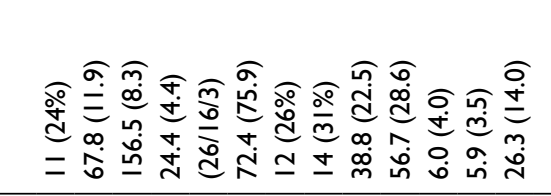 & 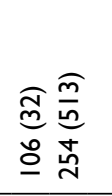 & 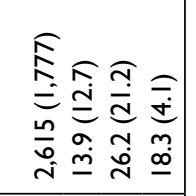 & 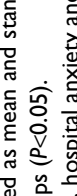 \\
\hline & & 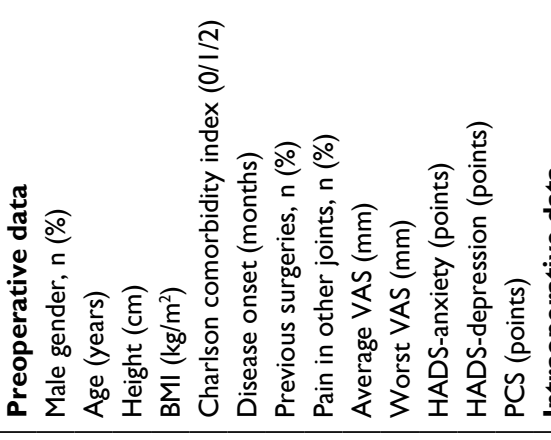 & 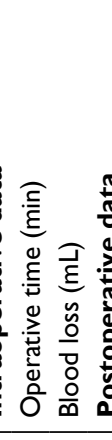 & 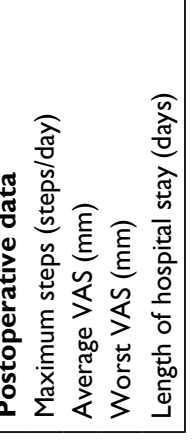 & 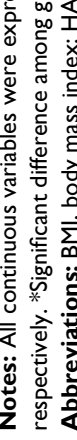 \\
\hline
\end{tabular}


Variation in physical activity is associated with old age. ${ }^{11,13}$ Similarly, in the present study, the age of the patients in the poor-pacing group was higher than that of the patients in the good-pacing group (poor-pacing group, 70.9 years; good-pacing group, 67.8 years). Patients with old age and vulnerability are carefully treated during variation in physical activity.

The amount of physical activity is important in predicting improvements in physical function after TKA. ${ }^{8}$ The present study showed that patients in the good-pacing group had significantly higher maximum steps per day and shorter duration of postoperative hospital stay than those in the poor-pacing group. Postoperative patients are encouraged to increase physical activity, because it results in better bone quality, enhancement of coordination and muscle strength, and, consequently, lower risk of falls. ${ }^{9}$ The amount of physical activity improves postoperative physical function. ${ }^{8,9}$ The present study showed that variation in physical activity throughout the day also has an impact in postoperative patients.

Severe postoperative pain is predicted by preoperative $\mathrm{PCS}^{25,26}$ and knee surgery. ${ }^{2}$ Pain catastrophizing is one of the fear-avoidance variables, which refers to the avoidance of movements or activities, based on fear. ${ }^{24}$ Treatment that incorporates a cognitive-behavioral intervention can lead to reduction in pain catastrophizing with a concurrent reduction in pain-related activity interference. ${ }^{27}$ Preoperative PCS showed no significant difference between the poor-pacing and good-pacing groups in the present study. Variation in physical activity was associated with postoperative pain, independent from preoperative $\mathrm{PCS}$, as shown in Table $\mathrm{S} 1$. The regulation of physical activity might be effective for pain even in the high pain catastrophizing patients.

\section{Limitations}

There are several limitations to this study. First, the present study investigated only the early postoperative period. The long-term effects of variation in physical activity on pain, disability, functional status, and quality of life have not been elucidated. Second, the effect of wearing and looking at the accelerometer has not been investigated. The accelerometer might increase the motivation of exercise and physical activity. Third, physical activity was measured using only the Lifecorder GS accelerometer. We cannot confirm that our results are consistent with those obtained using different motion counters. Fourth, all data were collected by researchers who were not blinded in this study. Fifth, the dose of analgesic medication was not recorded in detail. Finally, we included participants from a single medical center. Future studies using a longer follow-up period will be required to investigate the impact of variation in physical activity on the improvement of the pain, disability, functional status, and quality of life.

\section{Conclusion}

The present study showed that patients with variation in physical activity had severe postoperative pain and prolonged postoperative hospital stay. Desirable variation in physical activity in the postoperative period could improve the outcome in patients undergoing lower-limb arthroplasty.

\section{Acknowledgments}

The authors sincerely thank all of the patients, collaborating physicians, and other medical staff for their important contributions to this study. This work was supported, in part, by Grantsin-Aid for Scientific Research (no $15 \mathrm{H} 00683$ to K Hayashi) from the Ministry of Education, Culture, Sports, and Science and Technology of Japan.

\section{Disclosure}

The authors certify that no affiliation or financial involvement exists between them and any organization with a direct interest in the subject matter or materials discussed in the article, and report no conflicts of interest in this work.

\section{References}

1. Kurtz S, Ong K, Lau E, Mowat F, Halpern M. Projections of primary and revision hip and knee arthroplasty in the United States from 2005 to 2030. J Bone Joint Surg Am. 2007;89(4):780-785.

2. Pinto PR, Mcintyre T, Ferrero R, Almeida A, Araújo-Soares V. Risk factors for moderate and severe persistent pain in patients undergoing total knee and hip arthroplasty: a prospective predictive study. PLoS One. 2013;8(9):e73917.

3. Dahm KT, Brurberg KG, Jamtvedt G, Hagen KB. Advice to rest in bed versus advice to stay active for acute low-back pain and sciatica. Cochrane Database Syst Rev. 2010;6(6):CD007612.

4. Murphy SL, Lyden AK, Smith DM, Dong Q, Koliba JF. Effects of a tailored activity pacing intervention on pain and fatigue for adults with osteoarthritis. Am J Occup Ther. 2010;64(6):869-876.

5. Sharma V, Morgan PM, Cheng EY. Factors influencing early rehabilitation after THA: a systematic review. Clin Orthop Relat Res. 2009;467(6):1400-1411.

6. Pua YH, Ong PH. Association of early ambulation with length of stay and costs in total knee arthroplasty: retrospective cohort study. Am J Phys Med Rehabil. 2014;93(11):962-970.

7. Tayrose G, Newman D, Slover J, Jaffe F, Hunter T, Bosco J. Rapid mobilization decreases length-of-stay in joint replacement patients. Bull Hosp Jt Dis. 2013;71(3):222-226.

8. Taniguchi M, Sawano S, Kugo M, Maegawa S, Kawasaki T, Ichihashi N. Physical Activity Promotes Gait Improvement in Patients With Total Knee Arthroplasty. J Arthroplasty. 2016;31(5):984-988.

9. Paxton RJ, Melanson EL, Stevens-Lapsley JE, Christiansen CL. Physical activity after total knee arthroplasty: A critical review. World J Orthop. 2015;6(8):614-622. 
10. Andrews NE, Strong J, Meredith PJ. Activity pacing, avoidance, endurance, and associations with patient functioning in chronic pain: a systematic review and meta-analysis. Arch Phys Med Rehabil. 2012;93(11):2109-2121.

11. Andrews NE, Strong J, Meredith PJ. Overactivity in chronic pain: is it a valid construct? Pain. 2015;156(10):1991-2000.

12. Murphy SL, Smith DM, Lyden AK. Type of activity pacing instruction affects physical activity variability in adults with symptomatic knee or hip osteoarthritis. J Phys Act Health . 2012;9(3):360-366.

13. Kinkel S, Wollmerstedt N, Kleinhans JA, Hendrich C, Heisel C. Patient activity after total hip arthroplasty declines with advancing age. Clin Orthop Relat Res. 2009;467(8):2053-2058.

14. Taylor R. Interpretation of the correlation coefficient: a basic review. $J$ Diagn Med Sonogr. 1990;6(1):35-39.

15. Eberly LE. Correlation and simple linear regression. In: Walter T, editor Topics in Biostatistics (Methods in Molecular Biology). Ambrosius, Human Press Inc; 2007:143-64.

16. Charlson ME, Pompei P, Ales KL, Mackenzie CR. A new method of classifying prognostic comorbidity in longitudinal studies: development and validation. J Chronic Dis. 1987;40(5):373-383.

17. Schneider PL, Crouter SE, Lukajic O, Bassett DR. Accuracy and reliability of 10 pedometers for measuring steps over a 400-m walk. Med Sci Sports Exerc. 2003;35(10):1779-1784

18. Crouter SE, Schneider PL, Karabulut M, Bassett DR. Validity of 10 electronic pedometers for measuring steps, distance, and energy cost. Med Sci Sports Exerc. 2003;35(8):1455-1460.
19. Sullivan MJL, Bishop SR, Pivik J. The Pain Catastrophizing Scale: Development and validation. Psychol Assess. 1995;7(4):524-532.

20. Matsuoka H, Sakano Y. Assessment of cognitive aspect of pain: development reliability, and validation of Japanese version of Pain Catastrophizing Scale. Jpn J Psychosom Med. 2007;47:95-102.

21. Pallant JF, Bailey CM. Assessment of the structure of the Hospital Anxiety and Depression Scale in musculoskeletal patients. Health Qual Life Outcomes. 2005;3:82.

22. Matsudaira T, Igarashi H, Kikuchi H, et al. Factor structure of the Hospital Anxiety and Depression Scale in Japanese psychiatric outpatient and student populations. Health Qual Life Outcomes. 2009; 7:42.

23. Hasenbring MI, Verbunt JA. Fear-avoidance and endurance-related responses to pain: new models of behavior and their consequences for clinical practice. Clin J Pain. 2010;26(9):747-753.

24. Vlaeyen JW, Linton SJ. Fear-avoidance and its consequences in chronic musculoskeletal pain: a state of the art. Pain. 2000;85(3):317-332.

25. Riddle DL, Wade JB, Jiranek WA, Kong X. Preoperative pain catastrophizing predicts pain outcome after knee arthroplasty. Clin Orthop Relat Res. 2010;468(3):798-806.

26. Lunn TH, Gaarn-Larsen L, Kehlet H. Prediction of postoperative pain by preoperative pain response to heat stimulation in total knee arthroplasty. Pain. 2013;154(9):1878-1885.

27. Quartana PJ, Campbell CM, Edwards RR. Pain catastrophizing: a critical review. Expert Rev Neurother. 2009;9(5):745-758. 


\section{Supplementary material}

Table SI Multiple regression analysis for postoperative pain and length of hospital stay

\begin{tabular}{|c|c|c|c|c|c|c|}
\hline Dependent variables & Independent variables & B & SE & $\boldsymbol{\beta}$ & $p$-value & $\mathbf{R}^{2}$ \\
\hline \multirow[t]{3}{*}{ Postoperative VAS (average) } & Good-pacing/poor-pacing & 10.196 & 3.144 & 0.347 & 0.002 & 0.239 \\
\hline & Preoperative PCS & 0.305 & 0.121 & 0.270 & 0.014 & \\
\hline & Age & 0.322 & 0.147 & 0.237 & 0.032 & \\
\hline \multirow[t]{3}{*}{ Postoperative VAS (worst) } & Good-pacing/poor-pacing & 23.904 & 5.314 & 0.463 & $<0.001$ & 0.313 \\
\hline & Male & 22.438 & 6.704 & 0.345 & 0.001 & \\
\hline & TKA & $|1.26|$ & 5.077 & 0.224 & 0.030 & \\
\hline \multirow{2}{*}{$\begin{array}{l}\text { Length of postoperative } \\
\text { hospital stay }\end{array}$} & Good-pacing/poor-pacing & 3.017 & 1.064 & 0.310 & 0.006 & 0.424 \\
\hline & TKA & 2.587 & 1.037 & 0.272 & 0.015 & \\
\hline
\end{tabular}

Notes: The risk factors for sever postoperative pain and prolonged postoperative hospital stay were explored with multiple regression analysis in overall. The all preoperative and intraoperative variables were included in the multivariate logistic regression analysis. Sex, surgical procedure, and good-pacing/poor-pacing group included a dummy variable for analysis. The results showed the good-pacing/poor-pacing was a significant correlating factor for severe postoperative pain and prolonged postoperative hospital stay.

Abbreviations: $B$, nonstandard regression coefficient; SE, standard error; $\beta$, standardized regression coefficient; $\mathrm{R}^{2}$, multiple correlation coefficient adjusted for the degrees of freedom; VAS, visual analog scale; PCS, pain catastrophizing scale; TKA, total knee arthroplasty; HADS, hospital anxiety and depression scale.

\section{Publish your work in this journal}

The Journal of Pain Research is an international, peer reviewed, open access, online journal that welcomes laboratory and clinical findings in the fields of pain research and the prevention and management of pain. Original research, reviews, symposium reports, hypothesis formation and commentaries are all considered for publication.

\section{Dovepress}

The manuscript management system is completely online and includes a very quick and fair peer-review system, which is all easy to use. Visit http://www.dovepress.com/testimonials.php to read real quotes from published authors. 